\title{
ARTICLE
}

\section{The dependences of osteocyte network on bone compartment, age, and disease}

\author{
Xiaohan Lai ${ }^{1}$, Christopher Price ${ }^{2}$, Shannon Modla ${ }^{3}$, William R Thompson ${ }^{4}$, Jeffrey Caplan ${ }^{3}$, Catherine B Kirn-Safran ${ }^{5}$ \\ and Liyun Wang ${ }^{1}$
}

Osteocytes, the most abundant bone cells, form an interconnected network in the lacunar-canalicular pore system (LCS) buried within the mineralized matrix, which allows osteocytes to obtain nutrients from the blood supply, sense external mechanical signals, and communicate among themselves and with other cells on bone surfaces. In this study, we examined key features of the LCS network including the topological parameter and the detailed structure of individual connections and their variations in cortical and cancellous compartments, at different ages, and in two disease conditions with altered mechanosensing (perlecan deficiency and diabetes). LCS network showed both topological stability, in terms of conservation of connectivity among osteocyte lacunae (similar to the "nodes" in a computer network), and considerable variability the pericellular annular fluid gap surrounding lacunae and canaliculi (similar to the "bandwidth" of individual links in a computer network). Age, in the range of our study (15-32 weeks), affected only the pericellular fluid annulus in cortical bone but not in cancellous bone. Diabetes impacted the spacing of the lacunae, while the perlecan deficiency had a profound influence on the pericellular fluid annulus. The LCS network features play important roles in osteocyte signaling and regulation of bone growth and adaptation.

Bone Research (2015) 3, 15009; doi:10.1038/boneres.2015.9; Published online: 19 May 2015

\section{INTRODUCTION}

As the most abundant cells in bone, osteocytes form an extensive cellular network through numerous cell processes emanating from individual cell bodies. These cellular protrusions and cell bodies are housed with an extensive pore system, the lacunar-canalicular system (LCS), and buried within the bones mineralized matrix. This cellular network allows osteocytes to obtain nutrients from the blood supply, sense external mechanical signals, and communicate among themselves and with other cells on bone surfaces.' Previous experimental studies ${ }^{2-5}$ have demonstrated that osteocytes in intact bone change their metabolic activity rapidly after mechanical loading, indicating their function as mechanosensors. There is increasing evidence that osteocytes sense mechanical loading through the interstitial fluid flow around osteocyte cell membranes in the LCS. ${ }^{6-7}$ The spatial and temporal profiles of load-induced flow depend not only on the loading parameters but also the architecture of
LCS. Alterations to the LCS structures are expected to impact how osteocytes perceive external mechanical stimulation during the "outside-in" mechnosensing processes $^{8-10}$ by modulating the levels of stimulatory forces, such as fluid shear stresses ${ }^{9}$ and drag forces on the pericellular tethering fibers of osteocytes. ${ }^{11}$ In response to these cellular stimulations, osteocytes release many signaling molecules like nitric oxide (NO), adenosine triphosphate (ATP), sclerostin, prostaglandin E2 (PGE2), and osteoprotegerin/receptor activator for nuclear factor ligand (OPG/RANKL), which regulate osteoblastic bone formation as well as osteoclastic-targeted bone resorption. ${ }^{10,12-15}$. Because the principle intracellular transport mechanisms that enable these molecules to reach their target cells are diffusion and/or convection through the LCS, the LCS structure also plays an important role in osteocytes' "inside-out" signaling process. ${ }^{16-18}$ Using a mathematical model, ${ }^{19}$ we previously demonstrated that solute transport can be altered with varied LCS

'Department of Mechanical Engineering, University of Delaware, Newark, DE, USA; ${ }^{2}$ Biomedical Engineering Program, University of Delaware, Newark, DE, USA; ${ }^{3} \mathrm{DBI}$ Bioimaging Center, University of Delaware, Newark, DE, USA; ${ }^{4}$ Department of Physical Therapy, Indiana University, Indianapolis, IN, USA and ${ }^{5}$ Department of Biological Sciences, University of Delaware, Newark, DE, USA 
parameters. ${ }^{20}$ Furthermore, the surface area encasing the fluid-filled pericellular space in the LCS represents a significant interface for the regulation of mineral homeostasis. It is not surprising that LCS morphology has been recently shown to correlate with tissue mineralization. ${ }^{21-22}$

Because of its importance in bone physiology, the LCS morphology has been studied extensively using imaging tools with varied resolution $(20 \mu \mathrm{m}-1 \mathrm{~nm})$ and 2D- or 3D-imaging capability (see a recent comprehensive review $^{23}$ ). These studies provided quantitative assessments of the overall size, shape, volume fraction (porosity), and distribution density of the vascular channels, osteocyte lacunae, and canaliculi in different bones from many species. ${ }^{23}$ For example, lacunae were on the order of 290-455 $\mu^{3}$ (volume) and distributed at a number density of 26-90 lacunae per $\mathrm{mm}^{3}$ and lacunar separation of 21-40 $\mu \mathrm{m}$ from mouse to human bones. Canaliculi ranged from 95 to $553 \mathrm{~nm}$ in diameter and were distributed at 41-387 per lacuna for different species, with a mean matrix distribution of $\sim 0.55-0.85$ per $\mu \mathrm{m}^{2}$. Significant variations reported among these measures may be due to the different methodologies and subjects used in the studies, but they also likely reflect the dynamic nature of the LCS structure in normal and diseased conditions.

These data lead one to ask what the invariant and variant features of the osteocyte network are present in the adult skeleton of normal and diseased subjects. Similar to telecommunications or computer networks, the osteocyte network consists of multiple nodes (lacunae) and interconnecting links (canaliculi) of potentially varying bandwidth (i.e., capacities of signal transfer) among the nodes. As the telecommunication or computer networks allow digital signals to pass among nodes, LCS network's primary function is to allow osteocytes to obtain nutrients, sense mechanical loading through fluid flow, and communicate with other cells through molecular signaling. In this study, we focused on examining selective features of the osteocyte network in terms of the overall topology (such as the number density of canaliculi emanating from the lacunae) and detailed link bandwidth (such as the pericellular fluid annular gap). We quantified these network features in both cortical and cancellous compartments in long bones from younger and older adult mice as well as from mice with perlecan deficiency and in mice with diabetes, both of which exhibited altered responses to mechanical loading. ${ }^{11,24}$ Because the central role of osteocyte LCS in fluid flow and bone mechanosensing, their changes, if any, would be important to understand the pathology associated with two disease conditions (perlecan deficiency and diabetes). The objective of the present study was to identify the invariant and variant features of the osteocyte LCS network. This information will help understand the mechanisms for intercellular communication among bone cells as well as the roles of bone fluid flow in bone's response to mechanical forces, a potent anabolic factor regulating bone growth and adaptation.

\section{MATERIALS AND METHODS}

Experimental groups

Five groups of male mice ( $N=3$ mice in each group) of various ages and genotypes were used. Group 1 included adult, 15-week-old wild-type (WT) C57BL/6J mice (Jackson Laboratory), which served as younger controls to Group 2. Group 2 included 32-week-old C57BL/6J mice (older adults), which also served as WT controls to the perlecandeficient mice in Group 3. Group 3 consisted of 32-week-old C57BL/6J mice with homozygous C1532Yneo mutations in the PLN/HSPG2 gene. Initially developed to model human Schwartz-Jampel Syndrome, these mice (termed "Hypo" herein) exhibited a significant deficiency in perlecan expression ${ }^{25}$ and were bred back to C57BL/6J mice background in-house. Bones from Hypo mice were found to have altered canalicular structure ${ }^{26}$ and attenuated bone formation in response to tibial uniaxial loading ${ }^{11}$ in our previous studies. Group 4 consisted of 20-week-old Akita mice with heterozygous Ins2Akita mutation, a spontaneous type 1 diabetes model (Jackson Laboratory). Akita mice developed severe hyperglycemia from 5 weeks of age. Our previous work has shown that they failed to respond to anabolic ulnar loading when compared to age-matched WT mice. ${ }^{27}$ Group 5 included age-matched WT controls to Group 4 (20-week-old C57BL/6J). The University of Delaware Institutional Animal Care and Use Committee approved the handling and use of all animals in this study.

Confocal imaging and analysis of 3D osteocyte LCS macrostructure

Plastic embedded processing of basic fuchsinstained specimens. Immediately after sacrifice and dissection the right femora were prepared for basic fuchsin staining and plastic embedding following previously established protocols. ${ }^{28}$ Briefly, intact right femora were fixed in 10\% neutral buffered formalin for 24 hours at $4{ }^{\circ} \mathrm{C}$, repeatedly rinsed in phosphate-buffered saline, and bulk stained in $1 \%$ basic fuchsin dissolved in ethanol solutions with ascending concentrations $170 \%, 70 \%, 90 \%, 90 \%$, $100 \%, 100 \%$ ethanol v/v) with solution changes every 2-3 days. After clearance and embedding in methyl methacrylate, the distal one third of the femora were isolated and cut sagittally into $\sim 0.20-\mathrm{mm}$ thick slices using a lowspeed saw (Isomet, Buehler) and a diamond blade (Buehler). All sections were then polished to $\sim 0.15 \mathrm{~mm}$ in thickness using graded sandpapers of decreasing grit size (600, 800, 1200 grit; Buehler) and polishing liquids (6 \& 1- $\mu \mathrm{m}$; Buehler) to achieve a scratch-free finished surface. 
a

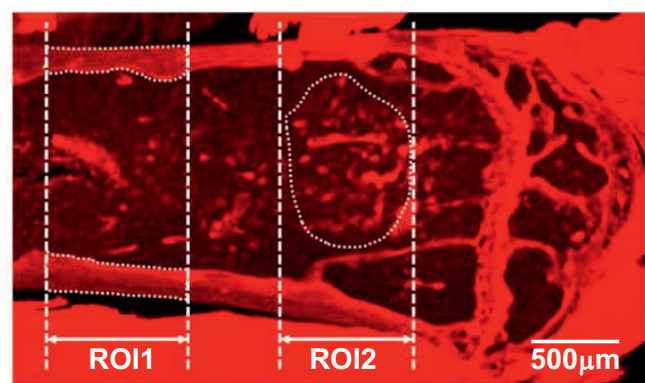

b
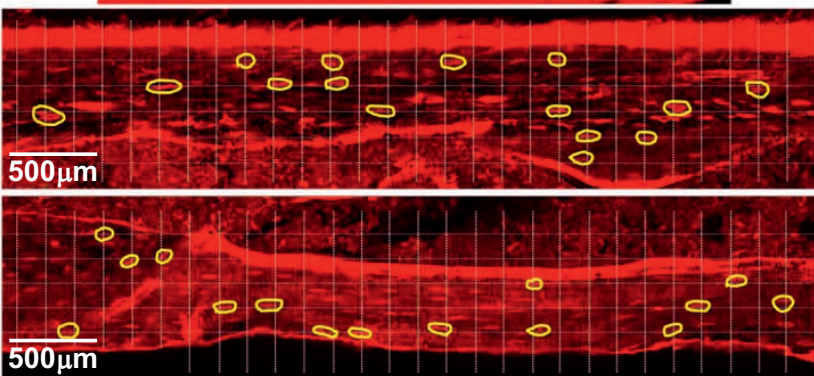

Figure 1. A representative confocal preview image (a) of basic fuchsin stained sagittal section of a murine distal femur, showing the two ROIs located in metaphyseal cortical and cancellous bone compartments. (b\&c) Due to larger number of lacunae in ROI1, a grid was overlaid on the image and 30-40 lacunae that fell on the inter-sections of the grid, as outlined by the yellow boundaries, were selected for a quick check of full-depth structures. Ten lacunae with intact 3D structures were chosen for high-resolution 3D imaging. For ROI2, ten lacunae were randomly chosen per animal without the grid.

The polished sections were then mounted on glass slides using Eukitt's mounting media and \# 1.5 glass coverglass.

Confocal imaging. Two regions of interests (ROI) were selected for study, including (i) femoral cortex and (ii) cancellous bone that were 1.5-2.15 mm, and 0.25-1 mm below the epiphyseal growth plate, respectively (Figure 1a). Ten lacunae were randomly selected for each ROI per animal. In the cortical ROI, a regularly spaced grid was placed atop a preview image (Figure $1 \mathrm{~b}$ and c) captured via an inverted confocal laser-scanning microscope (Zeiss LSM510, Carl Zeiss, Inc. NA., Thornwood, NY). Ten lacunae that fell on the grid intersections and showed intact full-depth structures under a quick z-stack imaging were selected for subsequent high-resolution 3D imaging. For the cancellous ROI, due to a smaller number of available lacunae, a selection grid was not utilized. Instead, 10 lacunae with intact full-depth structures were randomly chosen per animal from the region of cancellous bone. To quantify individual lacunae measurements, such as lacunar size and canalicular number density, z-stacks of high resolution, $2048 \times 2$ 048-pixel, images were captured for each of the selected lacunae and their canaliculi, utilizing the following optical parameters: excitation wavelength $=561 \mathrm{~nm}$, emission wavelength $=650 \mathrm{~nm}$, objective $=40 \times(1.2 \mathrm{NA})$ water lens (C-Apochromat W
Korr, Carl Zeiss, Inc. NA), pinhole $=1$ AU (optical slice thickness $=1.0 \mu \mathrm{m})$, zoom $=5$, pixel $=0.022 \mu \mathrm{m}$, scan speed $=$ $62 \mathrm{~s} /$ frame, frame averaging $=8, z$-step $=200 \mathrm{~nm}$. Detector gains and offsets were adjusted to maximize the dynamic range within a given image stack and a linear gain/offset compensation protocol was employed to insure consistent image signal/quality as individual z-stack scans imaged deeper into the tissue blocks. For measurements of lacuna density, individual $2048 \times 2$ 048-pixel frames were randomly captured throughout the tissue regions using a $10 \times(0.20 \mathrm{NA})$ objective and similar imaging settings as described above $(0.44 \mu \mathrm{m}$ per pixel). For groups 4 (Akita mice) and 5 (WT controls), only metaphyseal cortices were examined.

Quantitative measures of 3D LCS macrostructure. Threedimensional renderings of individual lacuna and their associated canaliculi were reconstructed from confocal z-stacks using the VOLOCITY software package (PerkinElmer). Using VOLOCITY, an initial selection and cropping of a ROI containing a single individual osteocyte lacuna and the emanating canaliculi was performed. Osteocyte lacunae were oriented such that the major/long axis of the osteocyte was aligned with the transverse (xy) plane of the image stack. The image stack was then filtered using an edge preserving smoothing filter to reduce the presence of noise in the image while maintaining structural integrity. 3D volume renderings of individual lacunae and their associated canaliculi were then generated (Figure 2a). Using these 3D renderings, the number of canaliculi emanating from the surface of each lacuna was counted directly. In order to calculate lacunae dimensions, individual lacunae were segmented from their associated canaliculi by outlining the lacunar body in each frame using the AMIRA software package (Visage Imaging, Inc., Figure 2b). Individual osteocyte lacunar volume, surface area, and the lengths of the major and minor axes of each ellipsoidal-shaped lacuna were measured in AMIRA. To correct for the distortion (stretching) of images in the axial (z) direction that is inherent in confocal microscopy, an axial correction factor of 0.803 was applied to the quantitative measures of rendered surfaces and volumes, which was experimentally measured for mineralized, basic fuchsinstained cortical bone using an established protocol. ${ }^{29}$ The number density of canaliculi emanating from an individual lacuna was calculated as the ratio of the total number of canaliculi and the lacunar surface area, and expressed as number per $\mathrm{mm}^{2}$. To calculate the number density of osteocyte lacuna in each bone region the number of lacunae within each $10 \times$ confocal image were counted and divided by the traced area of bone tissue in the same image and expressed as number per $\mathrm{mm}^{2}$. 
a

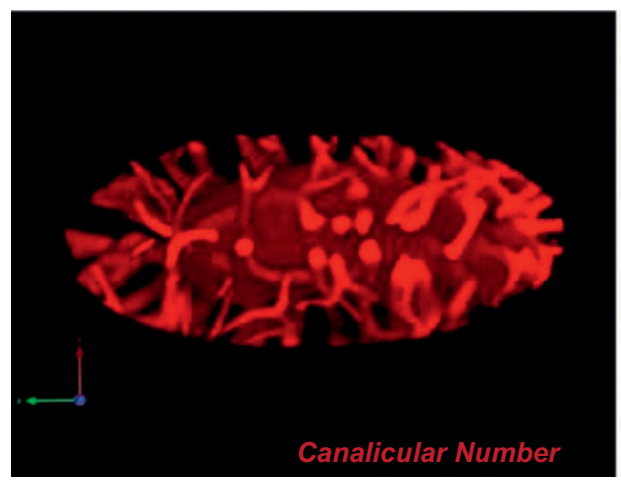

b

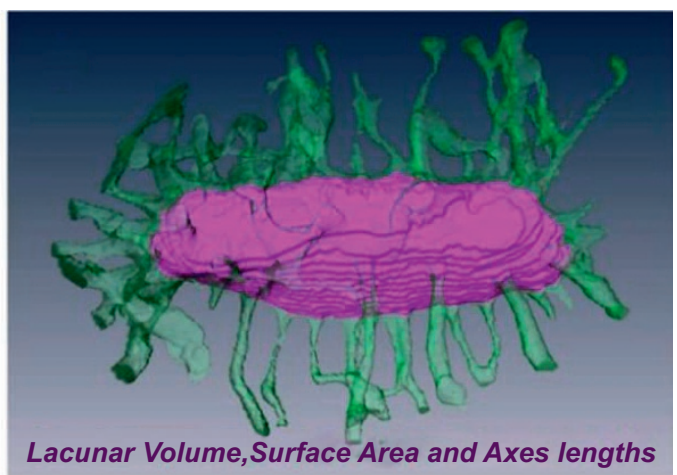

Figure 2. Representative 3D renderings built from z-stack confocal images of lacunae and associated canaliculi were used to quantify (a) canalicular number in VOLOCITY ${ }^{\circledR}$ and (b) lacunar volume, surface area and major and minor radii in AMIRA ${ }^{\circledR}$.

TEM analysis of osteocyte LCS ultrastructure

Processing for TEM imaging. Immediately after harvesting left metaphyseal femora were cut into small segments, each $\sim 1 \mathrm{~mm}$ in height, using a low-speed saw (Isomet, Buehler) and a diamond wafering blade. In order to preserve the cellular and pericellular structure of the LCS, femoral bone segments were immersion fixed in cold $\left(4{ }^{\circ} \mathrm{C}\right)$ fixative containing $4 \%$ paraformaldehyde, $2 \%$ glutaraldehyde, $0.7 \%$ ruthenium III hexamine trichloride (RHT) in $0.05 \mathrm{~mol}^{\circ} \mathrm{L}^{-1}$ cacodylate buffer $(\mathrm{pH} 7.5)$ for 24 hours, as described previously. ${ }^{30}$ Bone samples were then decalcified in $10 \%$ EDTA containing $1 \%$ paraformaldehyde in $0.1 \mathrm{~mol} \cdot \mathrm{L}^{-1}$ Tris-HCl buffer ( $\mathrm{pH}$ 7.4) for 14 days. Decalcification was performed at $4{ }^{\circ} \mathrm{C}$; the decalcification solution was refreshed every 2 days. After decalcification the samples were repeatedly washed in $0.1 \mathrm{~mol} \cdot \mathrm{L}^{-1}$ sodium-cacodylate buffer, postfixed for 3 hours with $1 \%$ osmium tetroxide in 0.1 cacodylate buffer, and washed. The samples were dehydrated in an ascending series of acetones and infiltrated with ascending Quetol

651-NSA/n-butyl glycidyl ether mixtures before being embedded in 100\% Quetol 651-NSA resin and polymerized at $60{ }^{\circ} \mathrm{C}$, as described previously. ${ }^{26}$ After polymerization, resin blocks containing the bones/bone segments of interest were isolated using a low-speed saw and faced for thin sectioning. Most sample blocks containing femoral metaphyseal bone segments were faced for sagittal sectioning, except for samples of groups 4 and 5 , which were prepared using cross sections. Ultrathin sections $(60-70 \mathrm{~nm}$ per section) were then cut on an ultramicrotome (Ultracut E; Reichert-Jung) and collected onto 200-mesh formvar/carbon-coated copper grids for imaging. Prior to imaging sections were post-stained with a saturated solution of uranyl acetate in methanol, followed by Reynolds' lead citrate. ${ }^{26}$

Transmission electron microscopy. Bone sections were imaged using a Libra 120 TEM (Carl Zeiss, Inc. NA) operated at $120 \mathrm{kV}$, and images were collected with a Gatan
Ultrascan 1000 CCD camera. ${ }^{26}$ Low magnification images $(100 \times, 0.1 \mu \mathrm{m}$ pixel) were acquired to identify trabecular and cortical bone and to prevent re-imaging of osteocytes on separate sections. High-resolution images of osteocyte canaliculi/processes and lacuna/cell bodies were acquired at $6300 \times$ magnification $(1.70 \mathrm{~nm}$ per pixel). For imaging lacunae, which were much larger than canaliculi, multiple adjacent, non-overlapping, TEM images were captured using the Gatan Digital Micrograph software and stitched together to generate high-resolution montage images. All images were exported as uncompressed TIFF files.

Quantitative analysis of osteocyte LCS ultrastructure. LCS ultrastructure was quantified by manually tracing lacunae, osteocyte cell bodies, canaliculi, and osteocyte cell process boundaries using Adobe Photoshop (Photoshop CS4; Adobe) and a digitizing pen display (Wacom Version 6.1.4). The lacunar wall and cell membrane as well as canalicular wall and cell process membrane were clearly delineated in the images (Figure 3a). These traces were batch exported as TIFF images to the BoneJ plugin in the Image J software and the dimensions of lacunae and the pericellular fluid spaces between the mineralized matrix walls and the cell membranes were obtained using a local thickness method, alternatively known as a "bubble method," and the mean thickness of the spaces was reported (Figure 3b and c). Similar analysis was applied to transversely oriented canaliculi, defined as having a shape factor (length/width) less than 2.5, in order to avoid measurement of oblate sections that were indicative of obliquely or longitudinally oriented canaliculi (Figure 4).

\section{Statistical analysis}

Descriptive data are presented as mean \pm standard deviation. Measurements from individual animals within the same groups were pooled because no differences were found among individual animals using analysis of variance 


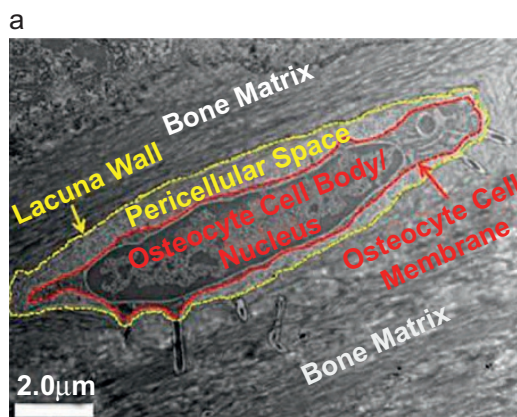

b

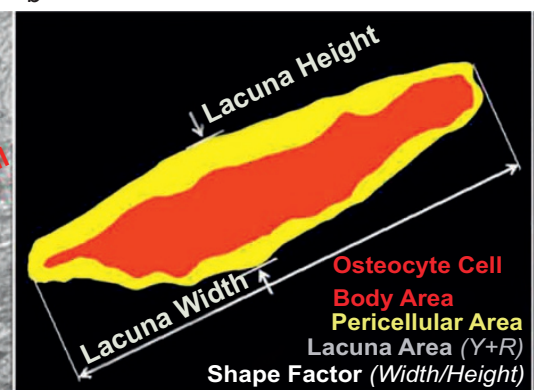

C

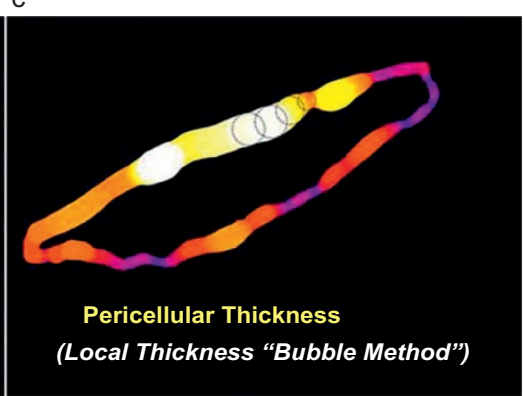

Figure 3. The ultrastructural measurements of ostecyte lacunae were obtained from TEM images by (a) tracing the lacunar wall and cell body in Photoshop ${ }^{\circledR}$ for $(\mathbf{b})$ quantifying the shape of the lacuna (width/height), the cross-sectional areas of lacuna, cell body, and the pericellular annulus in Image J. (c) The mean thickness of the pericellular annular gap was measured using the "bubble" method implemented in the BoneJ plugin of Image J.

(not shown). The total numbers of lacunae and canaliculi that were assessed for either confocal imaging or TEM analysis were reported. Unpaired Student's t-tests were performed to determine the differences between cortical and trabecular LCS parameters within each group. To determine the effects of age, perlecan deficiency, and diabetes on the LCS measurement outputs, unpaired two-sided Student's t-tests were performed between groups 1 and 2 ( 15 weeks vs. 32 weeks), between groups 3 and 2 (perlecan-deficient bones vs. WT), and between groups 4 and 5 (diabetic bones vs. WT), respectively. All tests were performed in GraphPad (San Diego, CA) with a significant level set at $P<0.05$.

\section{RESULTS}

Confocal-based measurements

The lacunar volume, surface area, major axis diameter, and density as well as canalicular number and number density were measured and compared.
Compartmental comparisons (cancellous vs. cortical). For the three groups of animals (C57BL/6J-15 weeks, C57BL/ 6J-32 weeks, and Hypo-32 weeks), there was a general trend that most measurements of lacunae and canalicular numbers were smaller than those in cortical bone (Table 1). However, only a few measures such as the major axis diameter of lacuna, lacunar density, and canalicular number reached statistical significance $(P<0.05$, Table 1$)$.

Effects of age (32 weeks vs. 15 weeks). Older adult mice (32 weeks) had significantly reduced $(8.1 \%, P=0.03$ ) lacunar density in cortical bone, whereas many other LCS measurements were not significantly different. It is noted that aging tended to decrease lacunar volume, lacunar surface area, lacunar density, canalicular number, and canalicular density in cancellous bone; yet, these changes did not reach significance (Table 2).

Effects of perlecan deficiency (Hypo vs. WT). All the LCS measurements showed varied levels of decrease in the a

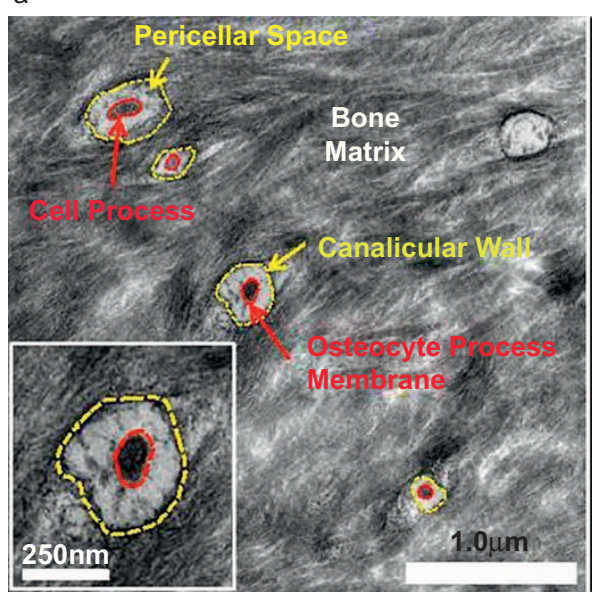

b

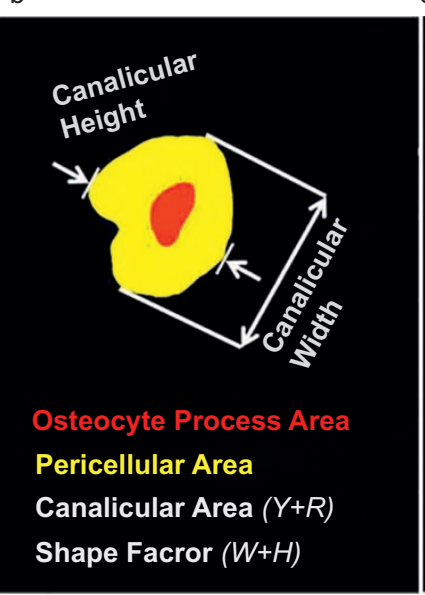

C

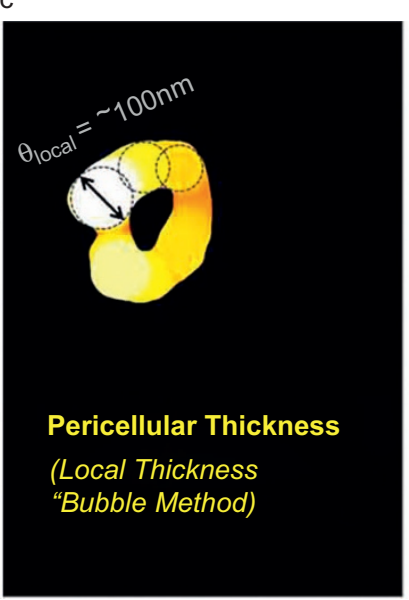

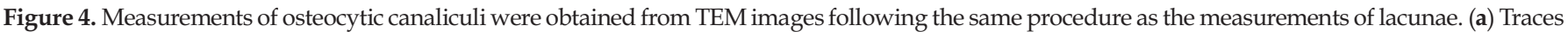

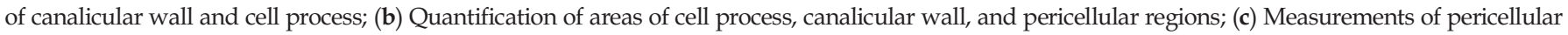
thickness in canaliculi using the "bubble" method. 
Table 1. Confocal-based LCS measurements-compartmental comparisons

\begin{tabular}{|c|c|c|c|c|c|c|c|c|c|}
\hline \multirow[b]{2}{*}{ Measurement/Unit } & \multicolumn{3}{|c|}{ C57BL/6J Mice (15 w) } & \multicolumn{3}{|c|}{ C57BL6J Mice (32 w) } & \multicolumn{3}{|c|}{ C1532Yneo perlecan hypomorph Mice (32 w) } \\
\hline & Cortical & Cancellous & Difference & Cortical & Cancellous & Difference & Cortical & Cancellous & Difference \\
\hline No. of lacuna. & 30 & 30 & & 30 & 30 & & 30 & 30 & \\
\hline Lacunar volume $/ \mu \mathrm{m}^{3}$ & $449.7 \pm 173.7$ & $400.9 \pm 133.1$ & $\begin{array}{l}-10.8 \% \\
P=0.23\end{array}$ & $447.2 \pm 209.4$ & $399.7 \pm 141.1$ & $\begin{array}{l}-10.7 \% \\
P=0.31\end{array}$ & $368.6 \pm 168.7$ & $305.4 \pm 150.4$ & $\begin{array}{l}-17.1 \% \\
P=0.13\end{array}$ \\
\hline $\begin{array}{l}\text { Lacunar surface } \\
\text { Area/ } \mu \mathrm{m}^{2}\end{array}$ & $410.0 \pm 108.0$ & $384.7 \pm 95.3$ & $\begin{array}{l}-6.3 \% \\
P=0.34\end{array}$ & $390.8 \pm 120.4$ & $350.4 \pm 88.0$ & $\begin{array}{l}-10.3 \% \\
P=0.14\end{array}$ & $347.7 \pm 97.4$ & $316.5 \pm 126.3$ & $\begin{array}{l}-8.9 \% \\
P=0.29\end{array}$ \\
\hline Lac. major axis/ $\mu \mathrm{m}$ & $15.8 \pm 2.9$ & $12.8 \pm 2.5$ & $\begin{array}{l}-18.9 \% \\
P<0.001\end{array}$ & $16.5 \pm 3.7$ & $13.6 \pm 3.6$ & $\begin{array}{l}-17.6 \% \\
P=0.003\end{array}$ & $15.4 \pm 3.7$ & $12.3 \pm 2.8$ & $\begin{array}{c}-20.1 \% \\
P=0.0005\end{array}$ \\
\hline Lacunar density $/ \mu \mathrm{m}^{-2}$ & $1200.7 \pm 149.0$ & $1078.2 \pm 213.9$ & $\begin{array}{l}-10.2 \% \\
P=0.01\end{array}$ & $1103.9 \pm 187.1$ & $1049.9 \pm 239.0$ & $\begin{array}{l}-4.9 \% \\
P=0.33\end{array}$ & $1075.5 \pm 185.3$ & $1010.5 \pm 253.7$ & $\begin{array}{l}-6.0 \% \\
P=0.26\end{array}$ \\
\hline Canalicular No. & $73.8 \pm 20.1$ & $68.3 \pm 13.0$ & $\begin{array}{l}-7.5 \% \\
P=0.21\end{array}$ & $77.5 \pm 12.0$ & $67.3 \pm 8.8$ & $\begin{array}{l}-13.2 \% \\
P<0.001\end{array}$ & $65.3 \pm 15.2$ & $59.3 \pm 17.2$ & $\begin{array}{l}-9.2 \% \\
P=0.16\end{array}$ \\
\hline $\begin{array}{l}\text { Canalicular No. } \\
\text { Density } / \mu \mathrm{m}^{-2}\end{array}$ & $0.209 \pm 0.048$ & $0.210 \pm 0.050$ & $\begin{array}{c}0.5 \% \\
P=0.94\end{array}$ & $0.209 \pm 0.043$ & $0.200 \pm 0.039$ & $\begin{array}{l}-4.3 \% \\
P=0.40\end{array}$ & $0.190 \pm 0.020$ & $0.190 \pm 0.020$ & $P>0.99$ \\
\hline
\end{tabular}

Note: Difference represented difference between compartments and it was calculated as (Cancellous-Cortical)/Cortical.

Hypo animals relative to the WT controls (Table 3). Canalicular number and canalicular number density were significantly reduced by $15.7 \%$ and $9.1 \%$ in the Hypo cortical bone compartment and lacunar volume and canalicular number showed $23.6 \%$ and $11.9 \%$ reduction in Hypo cancellous bone compartment (Table 3).

Effects of diabetes (Akita vs. WT). Although the LCS measures tended to be lower in diabetic Akita mice, only the lacunar density showed a significant decrease (10.1\%) relative to the non-diabetic WT controls (Table 4).

Summary of the results. A few compartment-, age-, and disease-associated changes in confocal-based LCS measures such as lacunar size, spacing, and their associated canaliculi were observed (Tables 1-4). Notably, perlecan deficiency and diabetes affected the LCS to different degrees. While perlecan deficiency resulted in significant decreases in more parameters (such as lacunar volume and the canalicular number density), diabetes impacted only the lacunar density. However, among all the examined parameters, the canalicular number density was highly consistent among all groups with mean values in the range of $0.190-0.210$ per $\mu \mathrm{m}^{2}$ and coefficients of variation in the range of $11-24 \%$. In comparison, the variations

Table 2. Confocal-based LCS measurements-effects of age

\begin{tabular}{lcc}
\hline & \multicolumn{2}{c}{ Difference/\%-(32 w-15 w)/15 w } \\
\cline { 2 - 3 } Measurement/Unit & Cortical & Cancellous \\
\hline Lacunar volume $/ \mu \mathrm{m}^{3}$ & $-0.6 \% P=0.96$ & $-0.3 \% P=0.97$ \\
Lacunar surface $\mathrm{area} / \mu^{2}$ & $-4.9 \% P=0.52$ & $-8.9 \% P=0.15$ \\
Lac. major axis $/ \mu \mathrm{m}$ & $4.2 \% P=0.42$ & $6.3 \% P=0.32$ \\
Lacunar density $/ \mu \mathrm{m}^{-2}$ & $-8.1 \% P=0.03$ & $-2.7 \% P=0.63$ \\
Canalicular No. & $5.0 \% P=0.39$ & $-1.5 \% P=0.73$ \\
Canalicular number density $/ \mu \mathrm{m}^{-2}$ & $0 P>0.99$ & $-4.8 \% P=0.39$ \\
\hline
\end{tabular}

Note: Comparisons were made between 15 weeks and 32 weeks WT male mice. seen in the size of lacunae were much higher (coefficients of variation in volume: $30-49 \%$ and in surface area: $21-40 \%$ ).

\section{TEM-based measurements}

The pericellular area and thickness in lacunae, the crosssectional areas of canalicular wall and cell process, as well as the pericellular area and thickness in canaliculi were measured and compared using TEM-based approaches. Please note that the numbers of lacunae and canaliculi examined under TEM may vary among groups. Although the numbers of animals, bone segments, and sections were the same for all the groups, we did not control the total bone areas and imaged all the available lacunae and canaliculi with good imaging quality.

Compartmental comparisons (cancellous vs. cortical). In contrast with the confocal-based measures, significant decreases were observed in the mean values of most measurements (with the exception of cell process crosssectional area) in the cancellous compartment compared with cortical bone for the three groups of animals (C57BL/6J-15 weeks, C57BL/6J-32weeks, and Hypo32 weeks) (Table 5). Cell process cross-sectional area was consistently greater in cancellous bone, and only

Table 3. Confocal-based LCS measurements-effects of perlecan deficiency

\begin{tabular}{lcr}
\hline & \multicolumn{2}{c}{ Difference/\%-(Hypo-WT)/WT } \\
\cline { 2 - 3 } Measurement/Unit & \multicolumn{1}{c}{ Cortical } & \multicolumn{1}{c}{ Cancellous } \\
\hline Lacunar volume $/ \mu \mathrm{m}^{3}$ & $-17.6 \% P=0.11$ & $-23.6 \% P=0.02$ \\
Lacunar surface $\mathrm{area} / \mu \mathrm{m}^{2}$ & $-11.0 \% P=0.13$ & $-9.7 \% P=0.23$ \\
Lac. major axis $/ \mu \mathrm{m}$ & $-6.7 \% P=0.25$ & $-9.6 \% P=0.12$ \\
Lacunar density $/ \mu \mathrm{m}^{-2}$ & $-2.5 \% P=0.56$ & $-3.8 \% P=0.54$ \\
Canalicular No. & $-15.7 \% P=0.001$ & $-11.9 \% P=0.03$ \\
Canalicular No. density $/ \mu \mathrm{m}^{-2}$ & $-9.1 \% P=0.03$ & $-5.0 \% P=0.22$ \\
\hline
\end{tabular}

Note: Comparisons were made between 32 weeks-old WT and perlecan deficient (Hypo) male mice. 
Table 4. Confocal-based LCS measurements-effects of diabetes

\begin{tabular}{|c|c|c|c|}
\hline \multirow[b]{2}{*}{ Measurement/Unit } & WT & Akita & \multirow{2}{*}{$\begin{array}{l}\text { Difference/\%- } \\
\text { (Akita-WT)/WT }\end{array}$} \\
\hline & Cortical & Cortical & \\
\hline No. of Lacuna & 30 & 30 & \\
\hline Lacunar volume/ $\mu \mathrm{m}^{3}$ & $462.4 \pm 177.7$ & $403.4 \pm 120.0$ & $-12.7 \% P=0.14$ \\
\hline Lacunar surface area $/ \mu \mathrm{m}^{2}$ & $449.4 \pm 129.5$ & $420.3 \pm 87.0$ & $-6.5 \% P=0.31$ \\
\hline 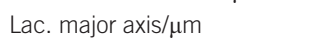 & $18.3 \pm 3.8$ & $18.2 \pm 2.8$ & $-0.55 \% P=0.91$ \\
\hline Lacunar density $/ \mu \mathrm{m}^{-2}$ & $1073.3 \pm 167.8$ & $965.1 \pm 132.6$ & $-10.1 \% P=0.008$ \\
\hline Canalicular No. & $93.4 \pm 23.1$ & $85.53 \pm 16.3$ & $-8.5 \% P=0.13$ \\
\hline Canalicular No. density $/ \mu \mathrm{m}^{-2}$ & $20.210 \pm 0.013$ & $0.205 \pm 0.022$ & $-2.4 \% P=0.29$ \\
\hline
\end{tabular}

Note: Measurements were made on 20-weeks-old C57BL/6J (WT) and C57BL/6Ins2Akita/J (Akita) male mice (three animals per group).

canalicular wall area remained unchanged in 15 week WT and 32 week Hypo mice ( $P>0.05$, Table 5).

Effects of age (32 weeks vs. 15 weeks). Again, in contrast with the confocal-based measures, all parameters (with the exception of lacunar pericellular thickness) showed significant changes in cortical bone from 32-week-old mice. In particular, the cortical canalicular measures were significantly larger at 32 weeks, while these measures remained unchanged in the cancellous bone compartment (Table 6).

Effects of perlecan deficiency (Hypo vs. WT). Perlecan deficiency reduced all the TEM-based measures with high statistical significance, except for the cell process area in cancellous bone (Table 7). The impact was broad and independent of the bone compartment.

Effects of diabetes (Akita vs. WT). Diabetes did not significantly alter the pericellular space in lacunae and only marginally within the canaliculi, while the canalicular wall area and cell process area were significantly reduced in diabetic cortical bone (Table 8). However, the alterations seen in diabetes were less dramatic than those seen in perlecan deficiency (Table 7).

Summary of the results. In comparison with the confocalbased measures, more extensive changes in the TEM-based measures were observed with different compartments, ages, and disease conditions. Among the factors examined herein, tissue compartment and perlecan deficiency showed greater effects on variations of the pericellular space than age and diabetes (Tables 5-8).

\section{DISCUSSION}

Measurements of the morphology of lacunae and canaliculi in five experimental animal groups presented comprehensive snap-shots of the dimensions of the osteocyte network, from which the invariant and variant features of the network were derived by comparisons.

The most revealing finding from this study is that the canalicular number density emanated from individual lacunae was highly consistent, regardless of the size and location of the lacunae, animal age, and the status of diabetes or perlecan deficiency (Tables 1-4). This suggests that, as an osteoblast differentiates from a surface lining cell to an osteocyte embedded in bone matrix, it is likely programed to incorporate into the pre-existing LCS network via a specified number of canaliculi, and the number is likely determined mainly by the surface area of the lacuna containing the cell body. The number of canalicular connections appears to be quite conserved $\left(\sim 1\right.$ canaliculus per $5 \mu \mathrm{m}^{2}$ lacunar surface) within the age range examined here. In contrast, the synapse connections found in neural networks are susceptible to age-related loss. ${ }^{31-33}$ Furthermore, we did not find any difference in canalicular number density between cancellous and cortical compartments, although

Table 5. TEM-based LCS measurements_compartmental comparisons

\begin{tabular}{|c|c|c|c|c|c|c|c|c|c|}
\hline \multirow[b]{2}{*}{ Measurement/Unit } & \multicolumn{3}{|c|}{ C57BL/6J Mice (15 w) } & \multicolumn{3}{|c|}{ C57BL/6J Mice (32 w) } & \multicolumn{3}{|c|}{ C1532Yneo perlecan hypomorph Mice (32 w) } \\
\hline & Cortical & Cancellous & Difference & Cortical & Cancellous & Difference & Cortical & Cancellous & Difference \\
\hline No. of lacuna & 36 & 32 & & 39 & 33 & & 39 & 24 & \\
\hline Lac. pericellualr Area/ $\mu \mathrm{m}^{2}$ & $9.28 \pm 1.17$ & $7.15 \pm 1.87$ & $\begin{array}{l}-22.9 \% \\
P<0.005\end{array}$ & $7.72 \pm 1.88$ & $5.27 \pm 1.77$ & $\begin{array}{l}-31.7 \% \\
P<0.005\end{array}$ & $6.85 \pm 1.85$ & $2.93 \pm 0.87$ & $\begin{array}{l}-57.2 \% \\
P<0.005\end{array}$ \\
\hline Lac. pericellular thickness $/ \mu \mathrm{m}$ & $0.49 \pm 0.15$ & $0.25 \pm 0.04$ & $\begin{array}{l}-48.9 \% \\
P<0.005\end{array}$ & $0.47 \pm 0.09$ & $0.33 \pm 0.12$ & $\begin{array}{l}-29.8 \% \\
P<0.005\end{array}$ & $0.41 \pm 0.09$ & $0.20 \pm 0.05$ & $\begin{array}{l}-51.2 \% \\
P<0.005\end{array}$ \\
\hline No. of canaliculi & 721 & 718 & & 506 & 334 & & 475 & 280 & \\
\hline Canalicular area $/ \mu \mathrm{m}^{2}$ & $0.071 \pm 0.029$ & $0.072 \pm 0.033$ & $\begin{array}{c}1.4 \% \\
P=0.54\end{array}$ & $0.081 \pm 0.040$ & $0.072 \pm 0.032$ & $\begin{array}{l}-11.1 \% \\
P<0.005\end{array}$ & $0.066 \pm 0.031$ & $0.062 \pm 0.035$ & $\begin{array}{l}-6.1 \% \\
P=0.10\end{array}$ \\
\hline Cell process area/ $\mu \mathrm{m}^{2}$ & $0.015 \pm 0.008$ & $0.021 \pm 0.012$ & $\begin{array}{c}40 \% \\
P<0.005\end{array}$ & $0.017 \pm 0.013$ & $0.021 \pm 0.013$ & $\begin{array}{c}23.5 \% \\
P<0.005\end{array}$ & $0.015 \pm 0.009$ & $0.022 \pm 0.016$ & $\begin{array}{c}46.7 \% \\
P<0.005\end{array}$ \\
\hline Can. pericellular area/ $\mu \mathrm{m}^{2}$ & $0.056 \pm 0.025$ & $0.052 \pm 0.025$ & $\begin{array}{c}-7.1 \% \\
P<0.005\end{array}$ & $0.065 \pm 0.034$ & $0.052 \pm 0.024$ & $\begin{array}{c}-20 \% \\
P<0.005\end{array}$ & $0.053 \pm 0.026$ & $0.041 \pm 0.024$ & $\begin{array}{l}-22.6 \% \\
P<0.005\end{array}$ \\
\hline Can. Pericellular thickness $/ \mu \mathrm{m}$ & $0.095 \pm 0.028$ & $0.084 \pm 0.027$ & $\begin{array}{l}-11.6 \% \\
P<0.005\end{array}$ & $0.101 \pm 0.032$ & $0.084 \pm 0.028$ & $\begin{array}{l}-16.8 \% \\
P<0.005\end{array}$ & $0.089 \pm 0.027$ & $0.066 \pm 0.026$ & $\begin{array}{l}-25.8 \% \\
P<0.005\end{array}$ \\
\hline
\end{tabular}

Note: Difference represented difference between compartments and it was calculated as (Cancellous-Cortical)/Cortical 
Table 6. TEM-based LCS measurements-effects of age

\begin{tabular}{lcc}
\hline & \multicolumn{2}{c}{ Difference/\%-(32 w-15 w)/15 w } \\
\cline { 2 - 3 } Measurement/Unit & Cortical & Cancellous \\
\hline Lac. pericellualr area/ $/ \mathrm{m}^{2}$ & $-16.8 \% P<0.005$ & $-26.3 \% P<0.005$ \\
Lac. pericellular thickness $/ \mu \mathrm{m}$ & $-4.1 \% P=0.48$ & $32 \% P<0.005$ \\
Canalicular area/ $\mu \mathrm{m}^{2}$ & $14.1 \% P<0.005$ & $0 P>0.99$ \\
Cell process area/ $\mu \mathrm{m}^{2}$ & $13.3 \% P<0.005$ & $0 P>0.99$ \\
Can. pericellular area/ $\mu \mathrm{m}^{2}$ & $16.1 \% P<0.005$ & $0 P>0.99$ \\
Can. pericellular thickness $/ \mu \mathrm{m}$ & $6.3 \% P<0.005$ & $0 P>0.99$ \\
\hline
\end{tabular}

Note: Comparisons were made between 15 weeks and 32 weeks WT male mice.

the average age of bone tissue (and hence the age of osteocytes) was likely younger within the cancellous compartment due to higher bone turnover. ${ }^{34}$ We did detect a change in lacunar shape (as shown in the longer major axis and thus more elongated osteocyte shape in cortical bone) among compartments, which was consistent with previous studies where the shape of osteocyte was found to depend on local stress distribution. ${ }^{35}$ As for the effects of diabetes on the LCS, we did not expect to see significant changes in the morphology of cell processes because the integrity of an osteocyte process is mainly dependent on actin and microtubules, ${ }^{36-37}$ which may not be altered in the presence of diabetes. However, the number density of lacunae in diabetic cortical bone was $10 \%$ lower than that in the normal age-matched mice, possibly due to impaired osteoblast differentiation commonly associated with diabetes. ${ }^{38-39}$ In the other disease condition examined in this study, a reduction in osteocyte canalicular density was found in bones from mice deficient in perlecan, an extracellular matrix heparan sulfate extracellular matrix proteoglycan. While it remains unclear how perlecan regulates canalicular density, these results are consistent with our previous findings. ${ }^{26}$ The consistent canalicular number density demonstrates that the topology of the overall canalicular connections is an invariant feature of the osteocyte network. This agrees with the notion that one of the major purposes of the extensive LCS network is to ensure the survival of osteocytes encased in mineralized

Table 7. TEM-based LCS measurements-effects of perlecan deficiency

\begin{tabular}{lcc}
\hline & \multicolumn{2}{c}{ Difference/\%- (Hypo-WT)/WT } \\
\cline { 2 - 3 } Measurement/Unit & \multicolumn{1}{c}{ Cortical } & Cancellous \\
\hline Lac. pericellualr area/ $\mu \mathrm{m}^{2}$ & $-11.3 \% P=0.04$ & $-44.4 \% P<0.005$ \\
Lac. pericellular thickness $/ \mu \mathrm{m}$ & $-12.8 \% P<0.005$ & $-39.4 \% P<0.005$ \\
Canalicular area/ $\mu \mathrm{m}^{2}$ & $-18.5 \% P<0.005$ & $-13.9 \% P<0.005$ \\
Cell process area $/ \mu \mathrm{m}^{2}$ & $-11.8 \% P=0.006$ & $4.7 \% P=0.39$ \\
Can. pericellular area/ $/ \mathrm{m}^{2}$ & $-18.5 \% P<0.005$ & $-21.2 \% P<0.005$ \\
Can. pericellular thickness $/ \mu \mathrm{m}$ & $-11.9 \% P<0.005$ & $-21.4 \% P<0.005$ \\
\hline
\end{tabular}

Note: Comparisons were made between 32 weeks-old WT and perlecan deficient (Hypo) male mice. matrix by facilitating the transport of nutrients and cell-cell signaling molecules through the network.

Although the architecture of the canaliculi branches, i.e., topology, of the LCS network appears to be very robust, the dimension of the pericellular space surrounding the osteocyte cell body and cell process, a critical parameter determining the overall permeability of fluid/solute transport in the LCS and analogous to the bandwidth of an individual network link, is quite sensitive to changes in age and the composition of the pericellular matrix (Tables 5-8). With increasing age, the gap of the pericellular space increases in cortical canaliculi, mainly due to an expansion of the canalicular wall that exceeds the enlargement of the cell process (Table 6). In young WT animals (15 weeks), larger pericellular gaps were found in the cortical compartment than the cancellous compartment. However, the compartmental difference disappeared in older animals (Table 5). Among all factors considered, perlecan deficiency is associated with the most profound changes in the pericellular space observed in this study the pericellular fluid annulus around bone lacunae and canaliculi of the perlecan-deficient bone decreased in both cortical and cancellous compartments (Table 7). This result agrees with our previous findings that perlecan is an essential component of the pericellular matrix, which maintains adequate fluid/solute transport pathway by possibly inhibiting mineralization of the pericellular space. ${ }^{26}$ In contrast, diabetes shows no effect on the pericellular space, while it does reduce the areas of the canalicular wall and cell process simultaneously (Table 8).

These data suggest that the osteocyte LCS is a network with relatively stable nodes (lacunae) connected by canaliculi with a consistent number density (Figure 5). While metabolic factors such as diabetes can influence the spacing of the lacunae, the overall structure of the network is robustly determined within a very narrow range of the number density of canaliculi branching out of individual lacunae $\left(\sim 1\right.$ canaliculi per $5 \mu \mathrm{m}^{2}$ lacunar surface in adult mice). Such conservation of canalicular number is likely achieved through genetic pre-programing to meet the metabolic requirements for osteocyte survival so that larger lacunae are wired with more canaliculi. Meanwhile, the LCS network is very dynamic, demonstrating significant variations and modifications on the pericellular fluid annulus around each canalicular connection or lacunar node (Figure 5). It becomes increasingly clear that osteocytes are capable of releasing osteolytic agents such as MMPs to modify their surroundings, ${ }^{40}$ especially under lactation, hibernation, or pregnancy due to the need of releasing mineral from the skeleton. ${ }^{41}$ Furthermore, our results show that proper composition of the secreted osteocyte pericellular matrix plays an important role in maintaining the pericellular 
Table 8. TEM-Based LCS Measurements_Effects of Diabetes

\begin{tabular}{|c|c|c|c|}
\hline & C57BL/6J WT mice (20 w) & C57BL/6-Ins2 $2^{\text {Akita } / J ~ A d u l t ~ A k i t a ~(20 ~ w) ~}$ & \\
\hline No. of lacuna & 68 & 69 & \\
\hline Lac. pericellular thickness/ $\mu \mathrm{m}$ & $0.23 \pm 0.14$ & $0.28 \pm 0.21$ & $21.7 \% P=0.10$ \\
\hline No. of Canaliculi & 206 & 211 & \\
\hline Canalicular area/ $\mu \mathrm{m}^{2}$ & $0.059 \pm 0.023$ & $0.054 \pm 0.019$ & $-8.5 \% P=0.02$ \\
\hline Can. pericellular thickness/ $\mu \mathrm{m}$ & $0.079 \pm 0.020$ & $0.078 \pm 0.018$ & $-1.3 \% P=0.59$ \\
\hline
\end{tabular}

Note: Measurements were made on 20-weeks-old C57BL/6J (WT) and C57BL/6-Ins2Akita/J (Akita) male mice (three animals per group).

fluid annulus, as shown by the profound changes in the perlecan-deficient LCS. Since aging induces catabolic and anabolic cellular changes, the age-related differences in the LCS morphology may be attributed to increased osteolytic and decreased anabolic activities in aged osteocytes. ${ }^{11,42-43}$

There were several limitations in the study. Artifacts such as tissue shrinking during TEM sample processing are always a concern in such quantitative studies. To minimize the potential artifacts, we sectioned the mouse femora into smaller segments for faster and better penetration of fixatives. The addition of RHT in the fixatives helped stabilize the pericellular matrix as well. ${ }^{27}$ We also excluded sections when the pericellular spaces around lacunae were devoid of pericellular matrix, indicating poor fixation and possible tissue shrinkage. Since the TEM measurements were performed on $2 \mathrm{D}$ sections, the orientation of the samples could impact the data, especially the overall shape and dimension of the lacunae and canaliculi. To minimize this effect, we sampled a large number canaliculi (211721) and excluded those with shape factor larger than 2.5. However, we should treat the data of lacunar pericellular area and thickness with caution, since they might be more susceptible to orientation artifacts. It is also noted that the confocal measurements using basic fuchsinstained samples reflected more on the structures of LCS pore system rather than those of osteocytes, because the dye could stain both the cellular components but also the pericellular space encased in the mineralized bone matrix. However, due to the submicron thickness of the pericellular space in lacunae and canaliculi, the measurements of lacunae should be very close to those of osteocytes. The other limitation was that the data were derived from a small sample size of animals (three mice per group). However, the numbers of osteocytes studied were much higher $(N=30)$ and we did not find any difference among individual mice within the same group. Additional limitation was the small span of age (15-32 weeks) investi-

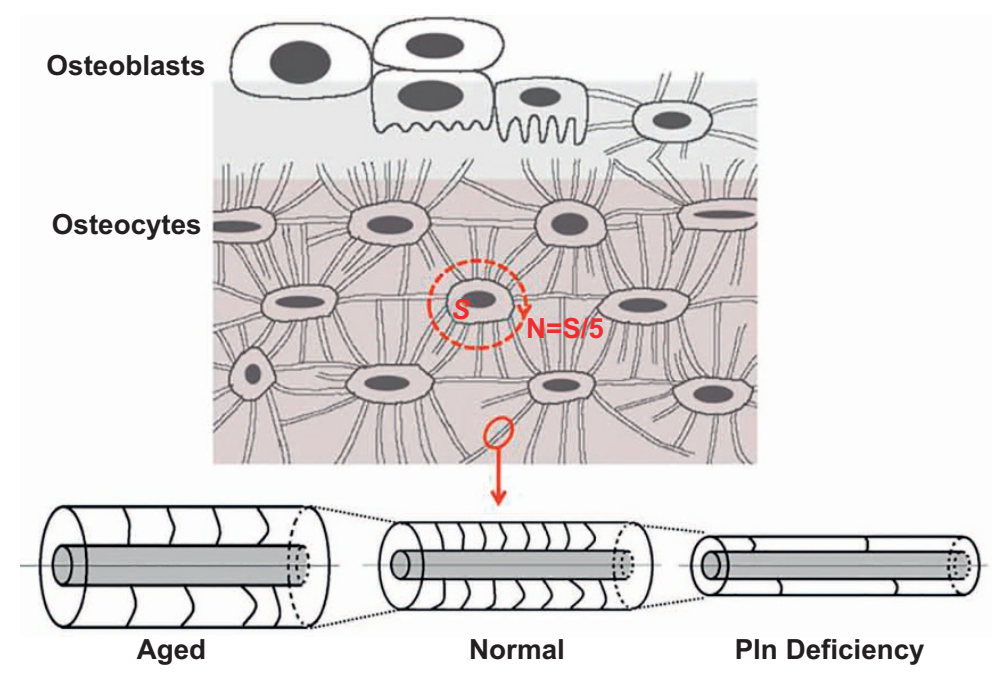

Figure 5. Osteoblasts are differentiated into osteocytes, which form an orderly network through the connecting canaliculi. The number of canaliculi is determined mainly by the surface area of lacuna $\left(N=S\left[\mu \mathrm{m}^{2}\right] / 5\right)$ regardless of bone compartment, age, and disease conditions. In cortical bone, aging is associated with larger canalicular annulus area and lower fiber density, while perlecan (Pln) deficiency reduces both annulus area and fiber density compared with wild types (Tables 6 and 7). 
gated herein. It would be better to know how LCS evolves during the entire developmental and aging process. Due to the technical limitation, all data have to be derived from individual time points and from different osteocyte populations. It would be ideal if the LCS network can be tracked longitudinally and quantified in the same cells. Advances in bioimaging in combination of transgenic mouse models where osteocytes are labeled with green fluorescent proteins could potentially make this feasible in the future.

In summary, key features such as the canalicular number density and pericellular fluid annular gap of the osteocyte lacunar-canalicular network were analyzed in trabecular and cortical compartments, in young and old bones, as well as from two diseased conditions (perlecan deficiency and diabetes). The LCS network showed both topological stability in terms of conserved numbers of connections, and considerable variability of "bandwidth" (communication capacity) of individual connections in terms of the pericellular annular fluid gap around lacunae and canaliculi. Our examination of diabetic cortex and perlecan-deficient bones revealed that the former condition mainly impact the spacing of the lacunae (the nodes in the LCS network), while the latter had profound influence in the pericellular fluid annulus (the connection's bandwidth). Age, in the range of our study (15-32 weeks) affected mainly the pericellular fluid annulus, possibly through altered osteolytic and anabolic activities in osteocytes. Quantifying the morphology and structure of the osteocyte lacunarcanalicular network will help enhance our understanding of osteocyte physiology and alterations associated with aging and skeletal disease conditions. The osteocyte LCS networking characteristics obtained in this study shed new insights onto not only the intercellular communication among bone cells but also the role of bone fluid flow in bone's response to mechanical forces, a potent anabolic factor regulating bone growth and adaptation.

\section{Competing interests}

The authors declare no conflict of interest.

\section{Acknowledgements}

This study was supported partially by funds from the NIH (RO1AR054385, P30GM103333).

\section{References}

1 Cowin SC. Bone Mechanics Handbook, 2nd edn. Boca Raton, FL: CRC Press, 2001.

2 Lean JM, Jagger CJ, Chambers TJ, Chow JW. Increased insulin-like growth factor I mRNA expression in rat osteocytes in response to mechanical stimulation. Am J Physiol 1995; 268: E318-E327.

3 Forwood MR, Kelly WL, Worth NF. Localisation of prostaglandin endoperoxide $\mathrm{H}$ synthase (PGHS)-1 and PGHS-2 in bone following mechanical loading in vivo. Anat Rec 1998; 252: 580-586.
4 Terai K, Takano-Yamamoto T, Ohba Y et al. Role of osteopontin in bone remodeling caused by mechanical stress. J Bone Miner Res 1999; 14: 839-849

5 Lee K, Jessop H, Suswillo R, Zaman G, Lanyon L. Endocrinology: bone adaptation requires oestrogen receptor-alpha. Nature 2003; 424: 389.

6 Price C, Zhou X, Li W, Wang L. Real-time measurement of solute transport within the lacunar-canalicular system of mechanically loaded bone: direct evidence for load-induced fluid flow. J Bone Miner Res 2011; 26: 277-285.

7 Thompson WR, Rubin CT, Rubin J. Mechanical regulation of signaling pathways in bone. Gene 2012; 503: 179-193.

8 Fritton SP, Weinbaum S. Fluid and solute transport in bone: flow-induced mechanotransduction. Annu Rev Fluid Mech 2009; 41: 347-374.

9 Weinbaum S, Cowin SC, Zeng Y. A model for the excitation of osteocytes by mechanical loading-induced bone fluid shear stresses. J Biomech 1994; 27: 339-360.

10 Ajubi NE, Klein-Nulend J, Nijweide PJ, Vrijheid-Lammers T, Alblas MJ, Burger EH. Pulsating fluid flow increases prostaglandin production by cultured chicken osteocytes - a cytoskeleton-dependent process. Biochem Biophys Res Commun 1996; 225: 62-68.

11 Wang B, Lai X, Price C et al. Perlecan-containing pericellular matrix regulates solute transport and mechanosensing within the osteocyte lacunar-canalicular system. J Bone Miner Res 2014; 29: 878-891.

12 Vezeridis PS, Semeins CM, Chen Q, Klein-Nulend J. Osteocytes subjected to pulsating fluid flow regulate osteoblast proliferation and differentiation. Biochem Biophys Res Commun 2006; 348: 1082-1088.

13 Tanaka K, Yamaguchi Y, Hakeda Y. Isolated chick osteocytes stimulate formation and bone-resorbing activity of osteoclast-like cells. J Bone Miner Metab 1995; 13: 61-70.

14 Heino TJ, Hentunen TA,Väänänen HK. Conditioned medium from osteocytes stimulates the proliferation of bone marrow mesenchymal stem cells and their differentiation into osteoblasts. Exp Cell Res 2004; 294: 458-468.

15 Thompson WR, Majid AS, Czymmek KJ et al. Association of the alpha(2)delta(1) subunit with $\mathrm{Ca}(\mathrm{v}) 3.2$ enhances membrane expression and regulates mechanically induced ATP release in MLO-Y4 osteocytes. J Bone Miner Res 2011; 26: 2125-2139.

16 Tami AE, Schaffler MB, Knothe Tate ML. Probing the tissue to subcellular level structure underlying bone's molecular sieving function. Biorheology 2003; 40: 577-590.

17 Wang B, Zhou X, Price C, Li W, Pan J, Wang L. Quantifying load-induced solute transport and solute-matrix interactions within the osteocyte lacunar-canalicular system. J Bone Miner Res 2013; 28: 1075-1086.

18 Li W, You L, Schaffler MB, Wang L. The dependency of solute diffusion on molecular weight and shape in intact bone. Bone 2009; 45: 1017-1023.

19 Zhou X, Novotny JE, Wang L. Modeling fluorescence recovery after photobleaching in loaded bone: potential applications in measuring fluid and solute transport in osteocytic lacunar-canalicular system. Ann Biomed Eng 2008; 36: 1961-1977.

20 Zhou X, Novotny JE, Wang L. Anatomic variations of the lacunarcanalicular system influence solute transport in bone. Bone 2009; 45: 704-710.

21 Kerschnitzki M, Kollmannsberger P, Burghammer M et al. Architecture of the osteocyte network correlates with bone material quality. J Bone Miner Res 2013; 28: 1837-1845.

22 Hesse B, Varga P, Langer M et al. Canalicular network morphology is the major determinant of the spatial distribution of mass density in human bone tissue: evidence by means of synchrotron radiation phase-contrast nano-CT. J Bone Miner Res 2015; 30: 346-356. 
23 Cardoso L, Fritton SP, Gailani G, Benalla M, Cowin SC. Advances in assessment of bone porosity, permeability and interstitial fluid flow. $J$ Biomech 2013; 46: 253-265.

24 Parajuli A, Gu X, Lai X, Zhang H, Thi MM, Price C. In vivo response of type I diabetic bone to mechanical loading depends on gender and/or disease severity. New Orleans, LA: Orthopaedic Research Society (ORS), 2014.

25 Rodgers KD, Sasaki T, Aszodi A, Jacenko O. Reduced perlecan in mice results in chondrodysplasia resembling Schwartz-Jampel syndrome. Hum Mol Genet 2007; 16: 515-528.

26 Thompson WR, Modla S, Grindel BJ et al. Perlecan/Hspg2 deficiency alters the pericellular space of the lacunocanalicular system surrounding osteocytic processes in cortical bone. J Bone Miner Res 2011; 26: 618-629.

27 Parajuli A, Li W, Gu X, Lai X, Price C, You L. Bone's responses to mechanical loading are impaired in type I diabetes, 2015. Bone In revision.

28 Burr DB, Hooser M. Alterations to the en bloc basic fuchsin staining protocol for the demonstration of microdamage produced in vivo. Bone 1995; 17: 431-433.

29 McCreadie BR, Hollister SJ, Schaffler MB, Goldstein SA. Osteocyte lacuna size and shape in women with and without osteoporotic fracture. J Biomech 2004; 37: 563-572.

30 You LD, Weinbaum S, Cowin SC, Schaffler MB. Ultrastructure of the osteocyte process and its pericellular matrix. Anat Rec A Discov Mol Cell Evol Biol 2004; 278: 505-513.

31 Adalbert R, Coleman MP. Axon pathology in age-related neurodegenerative disorders. Neuropathol Appl Neurobiol 2013; 39: 90-108.

32 Pakkenberg B, Gundersen HJ. Neocortical neuron number in humans: effect of sex and age. J Comp Neurol 1997; 384: 312-320.

33 Marner L, Nyengaard JR, Tang Y, Pakkenberg B. Marked loss of myelinated nerve fibers in the human brain with age. J Comp Neurol 2003; 462: 144-152.

34 Cowin S. Bone Mechanics Handbook, 2nd edn. Boca Raton, FL: CRC Press LLC, 2001, 1-27.
35 Vatsa A, Breuls RG, Semeins CM, Salmon PL, Smit TH, Klein-Nulend J. Osteocyte morphology in fibula and calvaria - is there a role for mechanosensing? Bone 2008; 43: 452-458.

36 Tanaka-Kamioka K, Kamioka H, Ris H, Lim SS. Osteocyte shape is dependent on actin filaments and osteocyte processes are unique actinrich projections. J Bone Miner Res 1998; 13: 1555-1568.

37 Murshid SA, Kamioka H, Ishihara Y, Ando R, Sugawara Y, TakanoYamamoto T. Actin and microtubule cytoskeletons of the processes of 3D-cultured MC3T3-E1 cells and osteocytes. J Bone Miner Metab 2007; 25: 151-158.

38 McCabe L, Zhang J, Raehtz S. Understanding the skeletal pathology of type 1 and 2 diabetes mellitus. Crit Rev Eukaryot Gene Expr 2011; 21: 187-206.

39 Motyl KJ, McCauley LK, McCabe LR. Amelioration of type I diabetesinduced osteoporosis by parathyroid hormone is associated with improved osteoblast survival. J Cell Physiol 2012; 227: 1326-1334.

40 Bonewald LF. The amazing osteocyte. J Bone Miner Res 2011; 26: 229-238.

41 Liu XS, Ardeshirpour L, VanHouten JN, Shane E, Wysolmerski JJ. Sitespecific changes in bone microarchitecture, mineralization, and stiffness during lactation and after weaning in mice. J Bone Miner Res 2012; 27: 865-875.

42 Busse B, Djonic D, Milovanovic P et al. Decrease in the osteocyte lacunar density accompanied by hypermineralized lacunar occlusion reveals failure and delay of remodeling in aged human bone. Aging Cell 2010; 9: 1065-1075. 43 Boskey AL, Coleman R. Aging and bone. J Dent Res 2010; 89: 1333-1348.

(c) (i) $९$ This work is licensed under a Creative Commons AttributionBY NC ND NonCommercial-NoDerivs 3.0 Unported License. The images or other third party material in this article are included in the article's Creative Commons license, unless indicated otherwise in the credit line; if the material is not included under the Creative Commons license, users will need to obtain permission from the license holder to reproduce the material. To view a copy of this license, visit http:/ / creativecommons.org/licenses/by-nc-nd/3.0/ 